\title{
A Useful Method for Deprotection of the Protective Allyl Group at the Anomeric Oxygen of Carbo- hydrate Moieties Using Tetrakis(triphenylphosphine)palladium
}

\author{
Kiyoshi Nakayama, Kouichi Uoto, Kunio Higashi, Tsunehiko Soga and Tsuneo Kusama* \\ Exploratory Research Laboratories I, Daiichi Pharmaceutical Co., Ltd., 16-13, Kitakasai 1-chome, Edogawa-ku, Tokyo 134, Japan. \\ Received December 3, 1991
}

\begin{abstract}
We have developed a simple method for the deprotection of allyl groups used as a protective group for the anomeric oxygen on a sugar moiety by employing tetrakis(triphenylphosphine) palladium in acetic acid. This method is applicable for not only simple but also complex allyl glycosides, such as natural lipid $A$ intermediates and other important lipid A intermediates. Further, this method would be useful for large-scale preparation.
\end{abstract}

Keywords deprotection; allyl group; tetrakis(triphenylphosphine)palladium; acetic acid; anomeric position; large-scale synthesis; one-pot reaction

In carbohydrate synthesis, the anomeric position is usually protected with suitable protecting groups such as benzyl, allyl or other groups. These must eventually be removed selectively and readily without affecting other functional groups on glycosides. In the course of synthesis of lipid A analogues, ${ }^{1,2)}$ the allyl group is the most useful protective group at the anomeric position of key intermediate compounds $\mathbf{2} \mathbf{a}^{1 b)}$ and $\mathbf{3 a},{ }^{2 c)}$ these being for the natural $E$. coli type or a less toxic analog (1). ${ }^{2 c)}$ Deprotection of the allyl group on compounds $\mathbf{2 a}$ and $\mathbf{3 a}$ has been performed with 1,5-cyclooctadienebis(methyldiphenylphosphine)iridium (I) hexafluorophosphate ([Ir(COD) $\left.\left.\left(\mathrm{PMePh}_{2}\right)_{2}\right] \mathrm{PF}_{6}\right){ }^{3)}$ one of the best reagents for this purpose. However, this reagent is not suitable for largescale preparation, because it is difficult to prepare in large quantities, and sodium hexachloroiridate (IV) used as the starting material is expensive. We therefore sought an effective and convenient deprotection reagent which could be used in large-scale synthesis of the above lipid A intermediates in place of $\left[\mathrm{Ir}(\mathrm{COD})\left(\mathrm{PMePh}_{2}\right)_{2}\right] \mathrm{PF}_{6}$.

General deprotection of allyl glycosides involves a two-step reaction as follows. First, the allyl group is

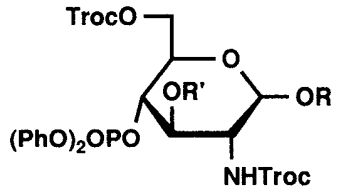

$$
\text { 2a: } \mathrm{R}=\mathrm{CH}_{2} \mathrm{CH}=\mathrm{CH}_{2}(\alpha)
$$$$
\text { 2b: } \mathbf{R}=\mathbf{H}(\alpha)
$$$$
\text { 9: } \mathbf{R}=\mathbf{A c}(\alpha)
$$$$
\mathbf{R}^{\prime}=\mathrm{COCH}_{2} \underset{\mathrm{OCO}\left(\mathrm{CH}_{2}\right)_{12} \mathrm{CH}_{3}}{\mathrm{CH}}\left(\mathrm{CH}_{2}\right)_{10} \mathrm{CH}_{3}
$$

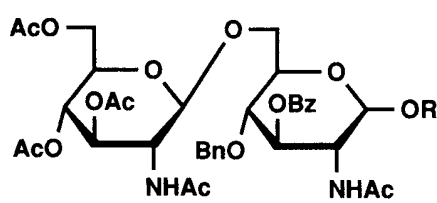

6a : $\mathbf{R}=\mathrm{CH}_{2} \mathrm{CH}=\mathrm{CH}_{2}(\beta)$

$6 b: R=H(\alpha)$

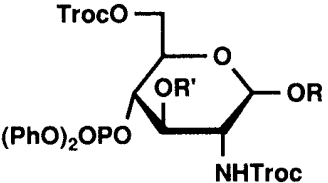

3a: $\mathbf{R}=\mathrm{CH}_{2} \mathrm{CH}=\mathrm{CH}_{2}(\alpha)$

3b: $\mathbf{R}=\mathbf{H}(\alpha)$

$R^{\prime}=\mathrm{COCH}_{2} \mathrm{NHCO}\left(\mathrm{CH}_{2}\right)_{10} \mathrm{CH}_{3}$

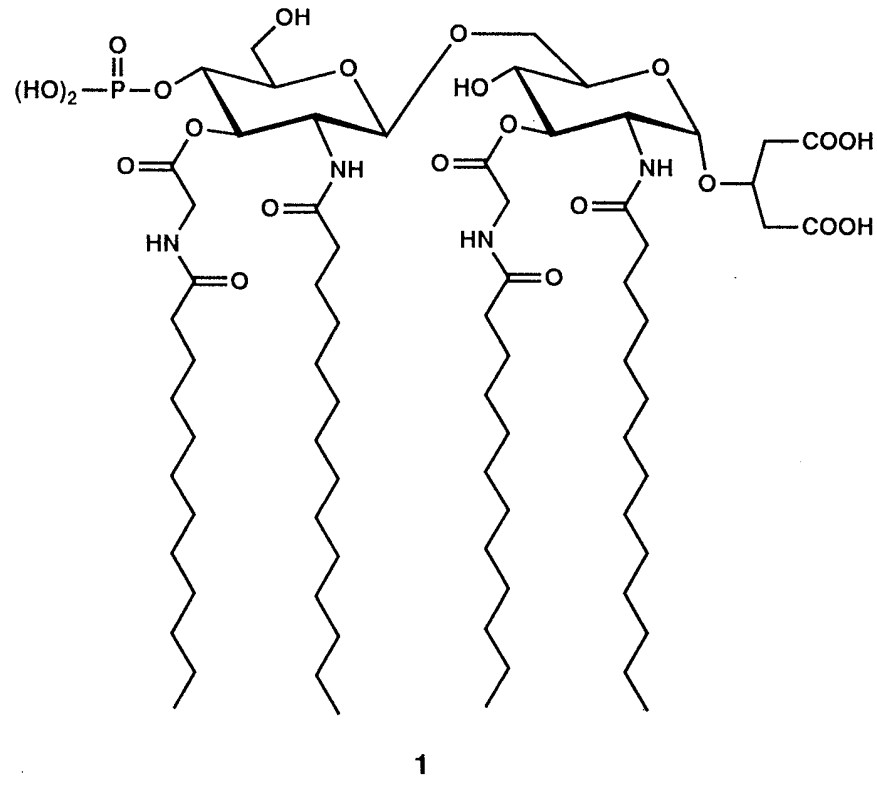

Fig. 1

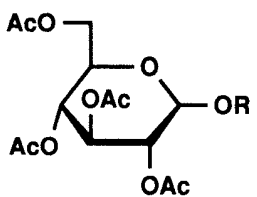

4a: $\mathbf{R}=\mathrm{CH}_{2} \mathrm{CH}=\mathrm{CH}_{2}(\beta)$ 4b: $R=H(\alpha)$

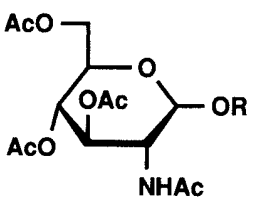

5a: $\mathbf{R}=\mathrm{CH}_{2} \mathrm{CH}=\mathrm{CH}_{2}(\beta)$ 5b: $\mathbf{R}=\mathbf{H}(\alpha)$

(R)

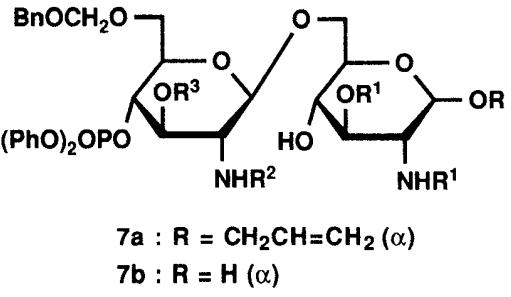

$R^{1}=\mathrm{COCH}_{2} \mathrm{CH}\left(\mathrm{CH}_{2}\right)_{10} \mathrm{CH}_{3}$

(R)

$R^{2}=\mathrm{COCH}_{2} \mathrm{CH}\left(\mathrm{CH}_{2}\right)_{10} \mathrm{CH}_{3}$

$\mathrm{OCO}\left(\mathrm{CH}_{2}\right)_{10} \mathrm{CH}_{3}$

(R)

$R^{3}=\mathrm{COCH}_{2} \mathrm{CH}\left(\mathrm{CH}_{2}\right)_{10} \mathrm{CH}_{3}$ OCO $\left(\mathrm{CH}_{2}\right)_{12} \mathrm{CH}_{3}$

Bn: benzyl

Bz: benzoy

Troc: 2,2,2-trichloroethoxycarbonyl

Fig. 2

(C) 1992 Pharmaceutical Society of Japan 
converted into a propenyl group by using a reagent such as tert-BuOK, ${ }^{4)} 10 \% \mathrm{Pd} / \mathrm{C},{ }^{5)} \mathrm{PdCl}_{2},{ }^{6)} \mathrm{RhCl}\left(\mathrm{PPh}_{3}\right)_{3},{ }^{\text {7) }}$ or $\left[\operatorname{Ir}(\mathrm{COD})\left(\mathrm{PMePh}_{2}\right)_{2}\right] \mathrm{PF}_{6}$. The propenyl group is then cleaved off by a reagent such as an acid, ${ }^{5,6)} \mathrm{HgCl}_{2}$ $\mathrm{HgO} / \mathrm{H}_{2} \mathrm{O},{ }^{8)}$ or $\mathrm{I}_{2} / \mathrm{H}_{2} \mathrm{O}^{9)}$ as shown in Chart 1 . Among these isomerization reagents, tert-BuOK is not suitable because it causes deacylation of the substrates. $\mathrm{RhCl}\left(\mathrm{PPh}_{3}\right)_{3}$ is said to be effective for simple glucosamine derivatives, ${ }^{10}$ ) but when it was applied to compound $\mathbf{2 a}$ or $\mathbf{3 a}$, the reactions did not proceed, regardless of the presence or absence of 1,4-diazabicyclo[2.2.2] octane. Another current method uses $\mathrm{Pd}$ reagents, commonly combining the reagent with an acid in a one-pot deprotection reaction. These reagents were found to be unable to cleave sufficient quantities of the allyl group of compound 3a. However, the desired reaction proceeded with a combination of $\mathrm{PdCl}_{2}$, sodium acetate $(\mathrm{AcONa})$ and aqueous acetic acid $(\mathrm{AcOH}),{ }^{6}$ to provide $3 \mathbf{b}$ in a yield of $11 \%$ with decomposition of the substrate. This was the key to development of the new method, giving the necessary mild, selective deprotection of the allyl group. We then examined various combinations of palladium reagent (Pd black, palladium acetate $\left(\mathrm{Pd}(\mathrm{OAc})_{2}\right)$, tetrakis(triphenylphosphine)palladium $\left.\left(\mathrm{Pd}\left(\mathrm{PPh}_{3}\right)_{4}\right)\right)$, and mild acids (Dcamphor-10-sulfonic acid (CSA), and $\mathrm{AcOH}$ ).

The results are shown in Table I. Pd black provided the deprotected product $\mathbf{3} \mathbf{b}$ in low yield, but as the reaction

deprotection of the allyl glycoside

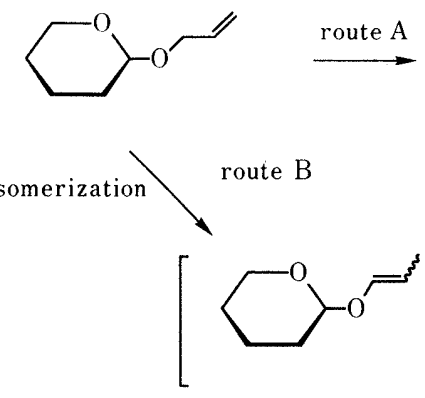

reagent
tert-BuOK
$\mathrm{Pd}$

$\mathrm{RhCl}\left(\mathrm{PPh}_{3}\right)_{3}$

$\left[\mathrm{Ir}(\mathrm{COD})\left(\mathrm{PMePh}_{2}\right)_{2}\right] \mathrm{PF}_{6}$
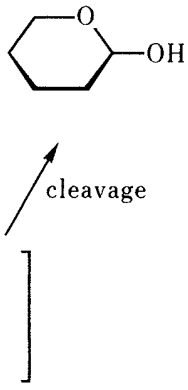

reagent

$\mathrm{H}^{+}$

$\mathrm{HgCl}_{2}, \mathrm{HgO} / \mathrm{H}_{2} \mathrm{O}$

$\mathrm{I}_{2} / \mathrm{H}_{2} \mathrm{O}$

Chart 1

TABle I. Deprotection of the Allyl Group of 3a Using Pd Reagent

\begin{tabular}{|c|c|c|c|c|c|c|c|}
\hline \multirow{2}{*}{$\begin{array}{c}\text { Run } \\
\frac{1}{1}\end{array}$} & \multirow{2}{*}{$\begin{array}{l}\text { Pd reagent } \\
(\text { eq) }\end{array}$} & \multirow{2}{*}{$\frac{\begin{array}{c}\text { Acid } \\
(\mathrm{eq})\end{array}}{\text { CSA (2) }}$} & \multirow{2}{*}{$\begin{array}{c}\text { Solvent } \\
\text { EtOH }\end{array}$} & \multirow{2}{*}{$\begin{array}{c}\begin{array}{c}\text { Additive } \\
\text { (eq) }\end{array} \\
-\end{array}$} & \multicolumn{2}{|c|}{ Conditions } & \multirow{2}{*}{ 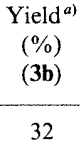 } \\
\hline & & & & & Reflux & $5 \mathrm{~h}$ & \\
\hline 2 & Pd black (5) & $\mathrm{AcOH}$ & $\mathrm{AcOH}$ & - & Reflux & $12 \mathrm{~h}$ & 5 \\
\hline 3 & Pd black (5) & CSA & THF & $\mathrm{PPh}_{3}(10)$ & Reflux & $10 \mathrm{~h}$ & - \\
\hline 4 & Pd black (5) & CSA & EtOH & $\mathrm{PPh}_{3}(10)$ & Reflux & $3 \mathrm{~h}$ & 51 \\
\hline $5^{b)}$ & Pd black (5) & $\mathrm{AcOH}$ & $\mathrm{AcOH}$ & $\mathrm{PPh}_{3}(10)$ & $80^{\circ} \mathrm{C}$ & $2 \mathrm{~h}$ & 71 \\
\hline $6^{b \xi}$ & Pd black (1) & $\mathrm{AcOH}$ & $\mathrm{AcOH}$ & $\mathrm{PPh}_{3}(2)$ & $80^{\circ} \mathrm{C}$ & $3 \mathrm{~h}$ & 50 \\
\hline $7^{b)}$ & $\mathrm{Pd}(\mathrm{OAc})_{2}(1)$ & $\mathrm{AcOH}$ & $\mathrm{AcOH}$ & $\mathrm{PPh}_{3}(4)$ & $80^{\circ} \mathrm{C}$ & $5 \mathrm{~h}$ & - \\
\hline $8^{b)}$ & $\mathrm{Pd}\left(\mathrm{PPh}_{3}\right)_{4}(1.0)$ & $\mathrm{AcOH}$ & $\mathrm{AcOH}$ & - & $80^{\circ} \mathrm{C}$ & $1 \mathrm{~h}$ & 88 \\
\hline $9^{b)}$ & $\mathrm{Pd}\left(\mathrm{PPh}_{3}\right)_{4}(0.5)$ & $\mathrm{AcOH}$ & $\mathrm{AcOH}$ & - & $80^{\circ} \mathrm{C}$ & $1 \mathrm{~h}$ & 86 \\
\hline $10^{b)}$ & $\mathrm{Pd}\left(\mathrm{PPh}_{3}\right)_{4}(0.3)$ & $\mathrm{AcOH}$ & $\mathrm{AcOH}$ & - & $80^{\circ} \mathrm{C}$ & $1 \mathrm{~h}$ & 79 \\
\hline $11^{b)}$ & $\mathrm{Pd}\left(\mathrm{PPh}_{3}\right)_{4}(0.1 \times 3)^{\mathrm{c})}$ & $\mathrm{AcOH}$ & $\mathrm{AcOH}$ & - & $80^{\circ} \mathrm{C}$ & $6 \mathrm{~h}$ & 75 \\
\hline $12^{b)}$ & $\mathrm{Pd}\left(\mathrm{PPh}_{3}\right)_{4}(0.1)$ & $\mathrm{AcOH}$ & $\mathrm{AcOH}$ & - & $80^{\circ} \mathrm{C}$ & $1 \mathrm{~h}$ & 21 \\
\hline
\end{tabular}

a) Isolated yield. b) Under a nitrogen atmosphere. c) $\mathrm{Pd}\left(\mathrm{PPh}_{3}\right)_{4}$ was added every $2 \mathrm{~h}$ after the first addition. time was lengthened to increase consumption of the starting material, the formation of several unidentified polar products increased (Table $I$, runs 1 and 2). When triphenylphosphine, a good ligand for $\mathrm{Pd}$ in $\mathrm{Pd}(0)$-catalyzed reactions, ${ }^{11)}$ was added to the reaction mixture, the yield of the deprotection product $\mathbf{3 b}$ was markedly increased (Table I, runs 4 and 5). Acetic acid was apparently the most effective acid and solvent in this reaction (Table I, runs 3-5). However, an excess amount of $\mathrm{Pd}$ and $\mathrm{PPh}_{3}$ was required to consume the starting material. A combination of only $1 \mathrm{eq}$ of $\mathrm{Pd}$ and $2 \mathrm{eq}$ of $\mathrm{PPh}_{3}$ actually decreased the yield of $\mathbf{3 b}$ (Table I, run 6). These results suggested that $\mathrm{Pd}(0)$ bound to $\mathrm{PPh}_{3}$ would prove to be an active species for the reaction. As we expected, $\mathrm{Pd}\left(\mathrm{PPh}_{3}\right)_{4}$ gave better results, requiring only $0.3 \mathrm{~mol}$ eq of the reagent and a short reaction time in a $40 \mathrm{~g}$ scale experiment (Table I, run 10 ).

Our results showed the $\mathrm{Pd}\left(\mathrm{PPh}_{3}\right)_{4} / \mathrm{AcOH}$ system to be a most promising method for the deprotection of allyl groups at the anomeric position. We therefore applied this method to other glycosides with allyl groups to examine its range of applicability and specificity. $\mathrm{Pd}\left(\mathrm{PPh}_{3}\right)_{4}$ successfully deprotected not only the glucosamine derivatives $\mathbf{5 a}^{12)}$ and $\left.6 \mathrm{a},{ }^{1 a}\right)$ known intermediates of a biosynthetic precursor of lipid $A$, but also the glucose derivative $\mathbf{4 a}^{13}$ ) (Table II, runs $1-3)$. The method was also applicable in the synthesis of natural $E$. coli-type lipid A to the key intermediates, $2 \mathbf{a},{ }^{1 b}$ ) and $7 \mathbf{a}^{1 b)}$ (Table II, runs 4 and 5).

Two pathways may operate in this reaction. One is via the propenyl glycoside described (route B) (Chart 1), and the other is by $\operatorname{Pd}(0)$ forming an $\pi$-allyl complex with the allyl glycoside, to eliminate pyranose (route A) (Chart 1). To ascertain which pathway actually operates, we investigated the stability of propenyl glycoside $\mathbf{8}$, synthesized with $\left[\operatorname{Ir}(\mathrm{COD})\left(\mathrm{PMePh}_{2}\right)_{2}\right] \mathrm{PF}_{6}$ from $\mathbf{3 a}$, in acetic acid. Heating compound 8 at $80^{\circ} \mathrm{C}$ in acetic acid for $12 \mathrm{~h}$ produced no reaction, and the starting material was recovered. It was also stable in the presence of $\mathrm{H}_{2} \mathrm{O}(10 \mathrm{eq})$ in acetic acid (Chart 2). If the deprotection reaction proceedded via the propenyl intermediate, compound 8 would be immediately converted into compound $\mathbf{3 b}$, because

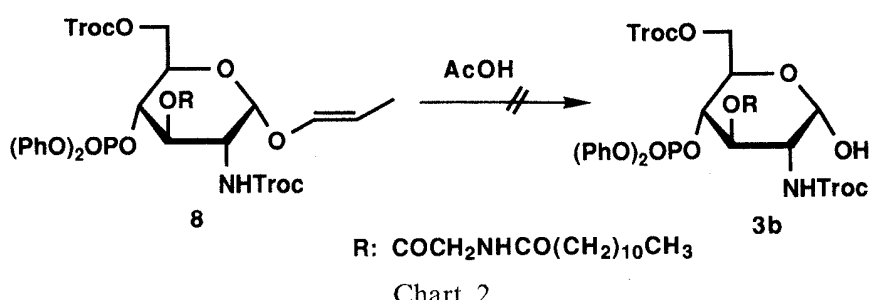

TABLE II. Deprotection of the Allyl Group Using $\mathrm{Pd}\left(\mathrm{PPh}_{3}\right)_{4} / \mathrm{AcOH}^{a)}$

\begin{tabular}{cccc}
\hline Run & Starting material & Reaction time & Yield (\%) \\
\hline 1 & $\mathbf{4 a}$ & $1 \mathrm{~h}$ & 78 \\
2 & $\mathbf{5 a}$ & $10 \mathrm{~min}$ & 96 \\
3 & $\mathbf{6 a}$ & $30 \mathrm{~min}$ & 98 \\
4 & $\mathbf{2 a}$ & $1 \mathrm{~h}$ & $92^{\mathbf{b}}$ \\
5 & $\mathbf{7 a}$ & $1 \mathrm{~h}$ & 72
\end{tabular}

a) All reactions were carried out using $0.3 \mathrm{~mol}$ eq of $\mathrm{Pd}\left(\mathrm{PPh}_{3}\right)_{4}$ in $\mathrm{AcOH}$ at $80^{\circ} \mathrm{C}$. b) The chemical structure was determined by leading the compound to the corresponding acetate (9). 
a reaction time of about $2 \mathrm{~h}$ or less was sufficient to complete the $\mathrm{Pd}\left(\mathrm{PPh}_{3}\right)_{4}$-catalyzed allyl deprotection reaction (Table I runs $8-12$, Table II). We therefore assumed that the reaction did not proceed via a propenyl intermediate, but by the formation of a $\pi$-allyl complex (route A) (Chart 1 ).

In summary, we have developed a new method for the deprotection of the allyl group at the anomeric position of carbohydrates. A unique feature of this method is the difference in its reaction mechanism from those of the known methods. We emphasize that the procedure is simple, being a one-pot reaction, which is mild and suitable for large-scale synthesis. This method would be therefore applicable in many areas of carbohydrate chemistry.

\section{Experimental}

Melting points were determined on a Yanagimoto melting point apparatus, and are uncorrected. Proton nuclear magnetic resonance $\left({ }^{1} \mathrm{H}-\mathrm{NMR}\right)$ spectra were obtained in deuteriochloroform on a JEOL GSX 500 spectrometer $(500 \mathrm{MHz})$. Chemical shifts are reported in parts per million relative to tetramethylsilane ( $\delta$ units) as an internal standard. Optical rotations were measured with a Horiba SEDA 200 polarimeter at $25^{\circ} \mathrm{C}$. Column chromatography was performed with Merck Silica gel 60 (70-230 mesh). Preparative thin-layer chromatography (preparative TLC) was performed by using silica gel (150A $1.0 \mathrm{~mm}$ thickness; PLK5F Whatman)

2-Deoxy-4-O-diphenylphosphono-3- $O$-( $N$-dodecanoylglycyl)-6- $O$-(2,2,2trichloroethoxycarbonyl)-2-(2,2,2-trichloroethoxycarbonylamino)- $\alpha$-Dglucopyranose (3b) (General Deprotection Procedure of the Allyl Group) $\mathrm{Pd}$ $\left(\mathrm{PPh}_{3}\right)_{4}(14.9 \mathrm{~g}, 12.9 \mathrm{mmol})$ was added to a solution of $\mathbf{3 a}(40.0 \mathrm{~g}$, $38.4 \mathrm{mmol})$ in acetic acid $(250 \mathrm{ml})$ under a nitrogen atmosphere at room temperature, and the mixture was heated at $80^{\circ} \mathrm{C}$ for $1 \mathrm{~h}$. The solvent was removed by azeotropic evaporation with toluene, and the residue purified by silica gel column chromatography $\left(\mathrm{CHCl}_{3}\right.$-acetone, $\left.40: 1-30: 1\right)$ to give $3 \mathbf{b}(30.2 \mathrm{~g}, 79 \%)$ as an oil. $[\alpha]_{\mathrm{D}}+31.0^{\circ}\left(c=1.0, \mathrm{CHCl}_{3}\right)$. Anal. Calcd for $\mathrm{C}_{38} \mathrm{H}_{49} \mathrm{Cl}_{6} \mathrm{~N}_{2} \mathrm{O}_{14} \mathrm{P}$ : C, 45.57; H, 4.93; N, 2.80. Found: C, 45.81; $\mathrm{H}$ 5.32; N, 2.73. ${ }^{1} \mathrm{H}-\mathrm{NMR} \delta: 0.88\left(3 \mathrm{H}, \mathrm{t}, J=7.3 \mathrm{~Hz}, \mathrm{CH}_{3}\right), 1.25(\mathrm{br}), 1.55$ $\left(2 \mathrm{H}, \mathrm{br}, \mathrm{CH}_{2} \mathrm{CH}_{2} \mathrm{CO}\right), 2.10\left(2 \mathrm{H}, \mathrm{t}, J=7.3 \mathrm{~Hz} \mathrm{CH} \mathrm{CH}_{2} \mathrm{CO}\right), 3.79(1 \mathrm{H}, \mathrm{dd}$ $\left.J=18.3,4.6 \mathrm{~Hz}, \mathrm{NCH}_{2} \mathrm{CO}\right), 3.92\left(1 \mathrm{H}, \mathrm{dd}, J=18.3,5.5 \mathrm{~Hz}, \mathrm{NCH}_{2} \mathrm{CO}\right)$ $4.08(1 \mathrm{H}$, td, $J=10.1,3.7 \mathrm{~Hz}, \mathrm{H}-2), 4.30(2 \mathrm{H}, \mathrm{m}, \mathrm{H}-5, \mathrm{H}-6), 4.47(1 \mathrm{H}, \mathrm{d}$ $J=10.1 \mathrm{~Hz}, \mathrm{H}-6^{\prime}$ ), $4.56,4.69$ (each $1 \mathrm{H}, \mathrm{AB}$ type d, $J=11.9 \mathrm{~Hz}, \mathrm{CH}_{2} \mathrm{CCl}_{3}$ ), $4.73\left(2 \mathrm{H}, \mathrm{d}, J=4.6 \mathrm{~Hz}, \mathrm{CH}_{2} \mathrm{CCl}_{3}\right), 4.78(1 \mathrm{H}, \mathrm{q}, J=10.1 \mathrm{~Hz}, \mathrm{H}-4), 5.32$ $(1 \mathrm{H}, \mathrm{d}, J=3.7 \mathrm{~Hz}, \mathrm{H}-1), 5.53(1 \mathrm{H}, \mathrm{t}, J=10.1 \mathrm{~Hz}, \mathrm{H}-3), 5.54(1 \mathrm{H}, \mathrm{d}$, $J=10.1 \mathrm{~Hz}, \mathrm{NH}), 6.28(1 \mathrm{H}, \mathrm{m}, \mathrm{NH}), 7.1-7.4(10 \mathrm{H}, \mathrm{m}$, arom. H).

2,3,4,6-Tetra- $\boldsymbol{O}$-acetyl- $\boldsymbol{\alpha}$-D-glucopyranose (4b) Compound $\mathbf{4 b}$ was prepared from $\mathbf{4 a}$ as described for the preparation of $\mathbf{3 b}$, with a yield of $78 \%$. 4b: a colorless crystalline solid. mp $109-111^{\circ} \mathrm{C}$. Anal. Calcd for $\mathrm{C}_{14} \mathrm{H}_{20} \mathrm{O}_{10}$ : C, 48.28; H, 5.79. Found: C, 48.35; H, 5.92. $[\alpha]_{\mathrm{D}}+136.0^{\circ}$ $\left(c=1.0, \mathrm{CHCl}_{3}\right),\left[\right.$ lit. $\left.^{14)}[\alpha]_{\mathrm{D}}+139^{\circ}\right]$.

2-Acetamido-2-deoxy-3,4,6-tri-O-acetyl- $\alpha$-D-glucopyranose (5b) Compound $\mathbf{5 b}$ was prepared from $\mathbf{5} \mathbf{a}$ as described for the preparation of $\mathbf{3 b}$, with a yield of $96 \%$. 5b: a colorless crystalline solid. mp $60-75^{\circ} \mathrm{C}$. Anal. Calcd for $\mathrm{C}_{14} \mathrm{H}_{21} \mathrm{NO}_{9}$ : C, 48.41; H, 6.09; N, 4.03. Found: $\mathrm{C}, 48.90 ; \mathrm{H}$, 6.15; N , 4.27. $[\alpha]_{\mathrm{D}}+48.9^{\circ}\left(c=1.0, \mathrm{CHCl}_{3}\right)$, [lit. $\left.{ }^{15)}[\alpha]_{\mathrm{D}}+49.4^{\circ}\right]$.

2-Acetamido-6- $O$-(2-acetamido-3,4,6-tri- $O$-acetyl-2-deoxy- $\beta$-D-glucopyranosyl)-3- $O$-benzoyl-4- $O$-benzyl-2-deoxy- $\alpha$-D-glucopyranose (6b) Compound $\mathbf{6 b}$ was prepared from $\mathbf{6} \mathbf{a}^{1 a)}$ as described for the preparation of $\mathbf{3 b}$, with a yield of $98 \%$. $6 \mathrm{~b}$ : a colorless crystalline solid. $\mathrm{mp} 231-234^{\circ} \mathrm{C}$ (dec.). Anal. Calcd for $\mathrm{C}_{36} \mathrm{H}_{44} \mathrm{~N}_{2} \mathrm{O}_{15}: \mathrm{C}, 58.06 ; \mathrm{H}, 5.96 ; \mathrm{N}, 3.76$. Found: $\mathrm{C}, 58.81 ; \mathrm{H}, 6.25 ; \mathrm{N}, 3.71 .[\alpha]_{\mathrm{D}}+8.1^{\circ}\left(c=1.7, \mathrm{CHCl}_{3}\right) .{ }^{1} \mathrm{H}-\mathrm{NMR} \delta: 1.85$ $\left(3 \mathrm{H}, \mathrm{d}, J=1.9 \mathrm{~Hz}, \mathrm{NCOCH}_{3}\right), 2.03\left(3 \mathrm{H}, \mathrm{s}, \mathrm{OCOCH}_{3}\right), 2.04(3 \mathrm{H}, \mathrm{d}$ $\left.J=1.9 \mathrm{~Hz}, \mathrm{NCOCH}_{3}\right), 2.07\left(3 \mathrm{H}, \mathrm{s}, \mathrm{OCOCH}_{3}\right), 2.10\left(3 \mathrm{H}, \mathrm{s}, \mathrm{OCOCH}_{3}\right)$, $3.44-3.56\left(2 \mathrm{H}, \mathrm{m}, \mathrm{H}-2^{\prime}, \mathrm{H}-4^{\prime}\right), 3.73\left(1 \mathrm{H}, \mathrm{m}, \mathrm{H}-5^{\prime}\right), 4.02(1 \mathrm{H}, \mathrm{d}, J=11.9 \mathrm{~Hz}$ $\left.\mathrm{H}-6^{\prime}\right), 4.17\left(2 \mathrm{H}, \mathrm{m}, \mathrm{CH}_{2} \mathrm{Ph}\right), 4.26\left(1 \mathrm{H}, \mathrm{dd}, J=11.9,4.6 \mathrm{~Hz}, \mathrm{H}-6^{\prime}\right), 4.31$ $(2 \mathrm{H}, \mathrm{m}, \mathrm{H}-2, \mathrm{H}-5), 4.51(1 \mathrm{H}, \mathrm{m}, \mathrm{H}-6), 4.59(1 \mathrm{H}, \mathrm{m}, \mathrm{H}-6), 5.05(2 \mathrm{H}, \mathrm{m}$, $\left.\mathrm{H}-4^{\prime}, \mathrm{OH}\right), 5.24(1 \mathrm{H}, \mathrm{d}, J=3.4 \mathrm{~Hz}, \mathrm{H}-1), 5.37\left(1 \mathrm{H}, \mathrm{d}, J=8.3 \mathrm{~Hz}, \mathrm{H}-1^{\prime}\right)$ $5.49\left(1 \mathrm{H}, \mathrm{t}, J=10.1 \mathrm{~Hz}, \mathrm{H}-3^{\prime}\right), 5.63(1 \mathrm{H}, \mathrm{t}, J=10.1 \mathrm{~Hz}, \mathrm{H}-3), 5.87(1 \mathrm{H}, \mathrm{m}$, $\mathrm{NH}), 5.97(1 \mathrm{H}, \mathrm{d}, J=9.2 \mathrm{~Hz}, \mathrm{NH}), 7.10(2 \mathrm{H}, \mathrm{m}, \operatorname{arom} . \mathrm{H}), 7.20(3 \mathrm{H}, \mathrm{m}$ arom. H), $7.45(2 \mathrm{H}, \mathrm{t}, J=7.3 \mathrm{~Hz}$, arom. $\mathrm{H}), 7.58(1 \mathrm{H}, \mathrm{t}, J=7.3 \mathrm{~Hz}$, arom. $\mathrm{H}), 8.03(1 \mathrm{H}, \mathrm{d}, J=7.3 \mathrm{~Hz}$, arom. $\mathrm{H})$.

1-O-Acetyl-2-deoxy-4-O-diphenylphosphono-3-O- $[(R)$-3-tetradecanoyloxytetradecanoyl]-6-O-(2,2,2-trichloroethoxycarbonyl)-2-(2,2,2trichloroethoxycarbonylamino)- $\alpha$-D-glucopyranose (9) Compound $2 \mathbf{b}$ was prepared from $2 \mathbf{a}^{1 b)}$ as described for the preparation of $\mathbf{3 b}$, with a yield of $92 \%$. The chemical structure of $\mathbf{2} \mathbf{b}$ was determined by leading it to the corresponding acetate 9. ${ }^{1 b)} 9$ : a colorless crystalline solid. mp $77-87^{\circ} \mathrm{C}$. Anal. Calcd for $\mathrm{C}_{54} \mathrm{H}_{78} \mathrm{Cl}_{6} \mathrm{NO}_{16} \mathrm{P}: \mathrm{C}, 52.27 ; \mathrm{H}, 6.34 ; \mathrm{N}, 1.13$. Found: $\mathrm{C}$, $52.11 ; \mathrm{H}, 6.35 ; \mathrm{N}, 1.42 .[\alpha]_{\mathrm{D}}+35.0^{\circ}\left(c=1.1, \mathrm{CHCl}_{3}\right),\left[\mathrm{lit}^{16)}[\alpha]_{\mathrm{D}}+35.6^{\circ}\right]$.

6-O-[6-O-Benzyloxymethyl-2-deoxy-4-O-diphenylphosphono-2-[(R)-3dodecanoyloxytetradecanoylamino $]-3-O-[(R)-3$-tetradecanoyloxytetradecanoyl $]-\beta$-D-glucopyranosyl $]-3-O-[(R)$-3-benzyloxytetradecanoyl] 2-[(R)-3-benzyloxytetradecanoylamino]-2-deoxy- $\alpha$-D-glucopyranose $(7 \mathbf{b})^{1 b)}$ $\mathrm{Pd}\left(\mathrm{PPh}_{3}\right)_{4}(32 \mathrm{mg}, 0.028 \mathrm{mmol})$ was added to a solution of $7 \mathbf{a}(200 \mathrm{mg}$, $0.090 \mathrm{mmol})$ in acetic acid $(2 \mathrm{ml})$ under a nitrogen atmosphere at room temperature, and the mixture was heated at $80^{\circ} \mathrm{C}$ for $1 \mathrm{~h}$. The solvent was removed by azeotropic evaporation with toluene, and the residue was diluted with AcOEt, washed with $5 \% \mathrm{NaHCO}_{3}$ and brine, dried over $\mathrm{MgSO}_{4}$, and concentrated in vacuo. The residue was purified by silica gel column chromatography $\left(\mathrm{CHCl}_{3}\right.$-acetone, $\left.20: 1\right)$ to give $7 \mathbf{b}(141 \mathrm{mg}, 72 \%)$ as an oil. $[\alpha]_{\mathrm{D}}+7.2^{\circ}\left(c=2.3, \mathrm{CHCl}_{3}\right) .{ }^{1} \mathrm{H}-\mathrm{NMR} \delta: 0.88(18 \mathrm{H}, \mathrm{t}, J=7.3 \mathrm{~Hz}$, $\left.\mathrm{CH}_{3} \times 6\right), 1.25(\mathrm{br}), 2.17-2.46(\mathrm{~m}), 2.60(1 \mathrm{H}, \mathrm{m}), 3.36(1 \mathrm{H}, \mathrm{t}, J=9.0 \mathrm{~Hz})$, $3.46(1 \mathrm{H}, \mathrm{m}), 3.60-3.88(\mathrm{~m}), 3.96-4.01(\mathrm{~m}), 4.18(1 \mathrm{H}, \mathrm{td}, J=10.0,3.4 \mathrm{~Hz}$, $\mathrm{H}-2), 4.48-4.63(\mathrm{~m}), 4.67-4.73(\mathrm{~m}), 5.08(1 \mathrm{H}, \mathrm{d}, J=3.4 \mathrm{~Hz}, \mathrm{H}-1), 5.13$ $(\mathrm{m}), 5.40(1 \mathrm{H}, \mathrm{d}, J=8.3 \mathrm{~Hz}), 5.51\left(1 \mathrm{H}, \mathrm{t}, J=10.0 \mathrm{~Hz}, \mathrm{H}-3^{\prime}\right), 6.27(1 \mathrm{H}, \mathrm{d}$, $J=9.3 \mathrm{~Hz}), 6.42(1 \mathrm{H}, \mathrm{d}, J=7.3 \mathrm{~Hz}), 7.12-7.36(25 \mathrm{H}, \mathrm{m}$, arom. H).

Propenyl 2-Deoxy-4-O-diphenylphosphono-3- $O$-( $\mathrm{N}$-dodecanoylglycyl)-6$O$-(2,2,2-trichloroethoxycarbonyl)-2-(2,2,2-trichloroethoxycarbonylamino)$\alpha$-D-glucopyranoside (8) Compound 8 was prepared from 3a by Shiba's method $^{1 b)}$ using Ir-complex. Compound $3 \mathrm{a}(1.50 \mathrm{~g}, 1.44 \mathrm{mmol})$ was reacted with Ir-complex $(30 \mathrm{mg})$ in tetrahydrofuran (THF) under nitrogen for $1.5 \mathrm{~h}$ at $50^{\circ} \mathrm{C}$ to afford $8(1.30 \mathrm{~g}, 87 \%)$ as an amorphous foam. Anal. Calcd for $\mathrm{C}_{41} \mathrm{H}_{53} \mathrm{Cl}_{6} \mathrm{~N}_{2} \mathrm{O}_{14} \mathrm{P}: \mathrm{C}, 47.28 ; \mathrm{H}, 5.13 ; \mathrm{N}, 2.69$. Found: C, 47.19; H, 5.25; $\mathrm{N}, 2.81 .[\alpha]_{\mathrm{D}}+51.6^{\circ}\left(c=1.0, \mathrm{CHCl}_{3}\right) .{ }^{1} \mathrm{H}-\mathrm{NMR} \delta: 0.88(3 \mathrm{H}, \mathrm{t}, J=7.3 \mathrm{~Hz}$, $\left.\mathrm{CH}_{3}\right), 1.25(\mathrm{br}), 1.58\left(3 \mathrm{H}, \mathrm{d}, J=5.4 \mathrm{~Hz}, \mathrm{CH}=\mathrm{CHCH}_{3}\right), 2.09(2 \mathrm{H}, \mathrm{t}$, $\left.J=7.3 \mathrm{~Hz}, \mathrm{CH}_{2} \mathrm{CO}\right), 3.77\left(1 \mathrm{H}, \mathrm{dd}, J=18.5,4.8 \mathrm{~Hz}, \mathrm{NCH}_{2} \mathrm{CO}\right), 3.92(1 \mathrm{H}$, $\left.\mathrm{dd}, J=18.5,5.4 \mathrm{~Hz}, \mathrm{NC}_{2} \mathrm{CO}\right), 4.05(1 \mathrm{H}, \mathrm{m}, \mathrm{H}-5), 4.13(1 \mathrm{H}, \mathrm{td}, J=10.3$, $3.4 \mathrm{~Hz}, \mathrm{H}-2), 4.30(1 \mathrm{H}, \mathrm{dd}, J=12.2,3.4 \mathrm{~Hz}, \mathrm{H}-6), 4.47(1 \mathrm{H}, \mathrm{d}, J=12.2 \mathrm{~Hz}$ $\left.\mathrm{H}-6^{\prime}\right), 4.56,4.68$ (each $1 \mathrm{H}, \mathrm{AB}$ type d, $\left.J=11.7 \mathrm{~Hz}, \mathrm{CH}_{2} \mathrm{CCl}_{3}\right), 4.73(2 \mathrm{H}$ s, $\left.\mathrm{CH}_{2} \mathrm{CCl}_{3}\right), 4.82(1 \mathrm{H}, \mathrm{q}, J=10.3 \mathrm{~Hz}, \mathrm{H}-4), 5.14(1 \mathrm{H}, \mathrm{d}, J=3.4 \mathrm{~Hz}, \mathrm{H}-1)$, $5.25(2 \mathrm{H}, \mathrm{m}, \mathrm{CH}=\mathrm{CH}), 5.48(1 \mathrm{H}, \mathrm{t}, J=10.3 \mathrm{~Hz}, \mathrm{H}-3), 7.1-7.4(10 \mathrm{H}, \mathrm{m}$, arom. $\mathrm{H})$

\section{References and Notes}

1) a) M. Imoto, H. Yoshimura, M. Yamamoto, T. Shimamoto, S Kusumoto and T. Shiba, Bull. Chem. Soc. Jpn., 60, 2197 (1987); b) M. Imoto, H. Yoshimura, T. Shimamoto, N. Sakaguchi, S. Kusumoto and T. Shiba, ibid., 60, 2205 (1987) and references cited therein.

2) a) T. Kusama, T. Soga, E. Shioya, K. Nakayama, H. Nakajima, Y Osada, Y. Ono, S. Kusumoto and T. Shiba, Chem. Pharm. Bull., 38, 3366 (1990); b) T. Kusama, T. Soga, Y. Ono, E. Kumazawa, E. Shioya, Y. Osada, S. Kusumoto and T. Shiba, ibid., 39, 1994 (1991); c) T. Kusama, T. Soga, Y. Ono, E. Kumazawa, E. Shioya, K. Nakayama, K. Uoto and Y. Osada, ibid., 39, 3244 (1991).

3) J. J. Oltvoort, C. A. A. van Boeckel, J. H. de Koning and J. H. van Boom, Synthesis, 1981, 305.

4) J. Gigg and R. Gigg, J. Chem. Soc. (C), 1966, 82.

5) R. Boss and R. Scheffold, Angew. Chem., 88, 578 (1976).

6) T. Ogawa, S. Nakabayashi and T. Kitazima, Carbohydr. Res. 114, 225 (1983).

7) P. A. Gent and R. Gigg, J. Chem. Soc., Chem. Commun., 1974, 277.

8) R. Gigg and C. D. Warren, J. Chem. Soc. (C), 1968, 1903.

9) M. A. Nashed and L. Anderson, J. Chem. Soc., Chem. Commun., 1982, 1274.

10) a) M. Inage, H. Chaki, S. Kusumoto and T. Shiba, Tetrahedron Lett., 21, 3889 (1980); b) M. Inage, H. Chaki, S. Kusumoto, T. Shiba, A. Tai, M. Nakahata, T. Harada and Y. Izumi, Chem. Lett., 1980, 1373; c) M. Inage, H. Chaki, S. Kusumoto and T. Shiba, Tetrahedron Lett., 22, 2281 (1981); d) S. Kusumoto, M. Imoto, T. Ogiku and T. Shiba, Bull. Chem. Soc. Jpn., 59, 1419 (1986).

11) a) B. M. Trost, Pure. Appl. Chem., 53, 2357 (1981); b) J. Tsuji, ibid. 58, 869 (1986), and references cited therein.

12) M. Kiso and L. Anderson, Carbohydr. Chem., 71, C12 (1979).

13) a) R. E. Lee and Y. C. Lee, Carbohydr. Res., 37, 193 (1974); b) T. Takano, F. Nakatsubo and K. Murakami, ibid., 203, 341 (1990).

14) F. Weygand, H. Ziemann and H. J. Bestmann, Chem. Ber., 91, 2534 (1958).

15) D. H. Leaback and P. G. Walker, J. Chem. Soc., 1957, 4754 